\title{
Membrane imaging by second-harmonic generation microscopy
}

\author{
L. Moreaux \\ Laboratoire de Neurophysiologie, Ecole Supérieure de Physique et Chimie Industrielles, Institut National de la \\ Santé et de la Recherche Médicale EP00-02, 10 rue Vauquelin, 75005 Paris, France
}

\section{O. Sandre}

Laboratoire Physico-Chimie, Institut Curie, Centre National de la Recherche Scientifique, Unité Mixte de Recherche 168, 11 rue Pierre et Marie Curie, 75005 Paris, France

\begin{abstract}
J. Mertz
Laboratoire de Neurophysiologie, Ecole Supérieure de Physique et Chimie Industrielles, Institut National de la Santé et de la Recherche Médicale EP00-02, 10 rue Vauquelin, 75005 Paris, France
\end{abstract}

Received February 24, 2000; revised manuscript received May 22, 2000

\begin{abstract}
We present a detailed analysis of the generation of second-harmonic radiation from biological membranes labeled with a styryl dye. In particular, we consider the high-numerical-aperture limit appropriate to highresolution microscopy in which an excitation beam is tightly focused from the side onto a membrane surface. In this limit the active surface area that contributes to second-harmonic generation (SHG) depends only on the tightness of the beam focus and the SHG radiation is confined by phase matching into two w ell-defined off-axis lobes. We derive expressions for the SHG radiation power, angular distribution, and polarization dependence in the cases of ideal or nonideal molecular alignment in the membrane and uniaxiality of the molecular hyperpolarizability. We define an SHG cross section similar to that used in two-photon-excited fluorescence (TPEF) to permit direct comparison of the two imaging modalities. Finally, we corroborate our results with experiments based on the excitation of a styryl dye in giant unilamellar vesicles with a mode-locked Ti:sapphire laser.
\end{abstract}

OCIS codes: 190.416, 190.4180, 180.5810, 190.4350, 190.4710.

\section{INTRODUCTION}

Nonlinear optics is proving to be a powerful tool for biological imaging. Fluorescence microscopy by two-photon excitation $^{1-3}$ has become a laboratory standard, and three-photon excitation has demonstrated its feasibility. ${ }^{4,5}$ More recently, nonlinear microscopies based on radiative harmonic generation have also been applied to the imaging of surfaces $^{6,7}$ and biological samples. Second-harmonic generation (SHG) has been used to image the intrinsic nonlinear susceptibility of muscle tissues ${ }^{8}$ and to image living cells labeled with styryl dyes. ${ }^{9,10}$ Third-harmonic generation has also been used to provide microscopic images of cells and plant samples. ${ }^{11,12}$ Harmonic generation is an optical phenomenon involving coherent radiative scattering, whereas fluorescence generation involves incoherent radiative absorption and reemission. As such, fluorescence and harmonic images are derived from fundamentally different contrast mechanisms. Moreover, because of its coherent nature, harmonic radiation is usually highly directional and depends critically on the spatial extent of the emission source, making a full description of harmonic generation more complicated than fluorescence generation. We limit our discussions here to the description of molecular SHG only, with the understanding that many of our techniques may be generalized to higher-order harmonics.
It is well known that SHG of dipolar origin cannot arise from a medium that possesses an inversion symmetry. Therefore molecular SHG is usually studied in geometries in which the radiating molecules are spatially ordered. A common geometry is that of a planar interface or a surface that imparts a preferential orientation to a thin layer of molecules. ${ }^{13}$ Typically, the layer is illuminated at an oblique angle by a laser beam, resulting in the emission of SHG beams in well-defined reflection and transmission directions. ${ }^{14,15}$ Because the laser beam is usually unfocused or weakly focused, both the driving laser field and the resultant SHG fields can be treated as simple collimated beams with well-defined wave vectors, and the layer of molecules is most appropriately described in this geometry by a macroscopic surface susceptibility that is independent of the illuminated surface area.

In contrast, if SHG is intended to provide microscopic image resolution, the driving laser beam must be focused to a small spot size. The driving field can no longer be considered a simple plane wave as above, and the structure of the resultant SHG radiation becomes critically dependent on the particular geometry of the driving field near the focal center. The use of a macroscopic surface susceptibility to quantify the SHG emission becomes inappropriate in this case, and one must resort to a more refined description of the surface nonlinearity for length 
scales much smaller than the radiation wavelength. In particular, it becomes necessary to redefine a surface nonlinearity starting from the level of individual molecular hyperpolarizabilities (or nonlinear molecular cross sections).

In this paper we provide a detailed theoretical description of SHG emission appropriate to a high-resolution scanning microscope configuration that uses a tightly focused excitation beam. The sensitivity of SHG emission to tight focusing has already been recognized for bulk nonlinear crystals. ${ }^{16}$ Because our attention is directed to biological imaging we consider here the different geometry of molecular SHG from membrane surfaces, as has been demonstrated experimentally in the imaging of cells and vesicles. ${ }^{10,17,18}$ We begin by characterizing the SHG emission from a single hyperpolarizable molecule in terms of a nonlinear scattering cross section. We then extend our discussion to the characterization of SHG emission from a planar distribution of molecules. In particular, we adopt a formalism that specifically permits a direct comparison between the radiated powers in the case of SHG and in the corresponding case of two-photonexcited fluorescence (TPEF); the latter is already well established in biological microscopy. Indeed, recent results show that simultaneous SHG and TPEF can be obtained from the same collections of molecules. Finally we corroborate our theoretical formalism with experiments based on a full characterization, in terms of power, angular distribution, and polarization, of the SHG radiation obtained from giant unilamellar vesicles labeled with styryl dye. Our research here serves as a follow-up to provide complete theoretical and experimental support for the results presented in Ref. 18 .

\section{THEORY OF SHG MICROSCOPY}

\section{A. Single Molecule}

We begin by defining the SHG cross section of a single molecule. The most straightforward definition entails calculating the total radiated SHG power and dividing this by the square of the incident driving field intensity. In particular, a molecule is considered an elemental dipole radiator driven by the excitation light according to its first hyperpolarizability. For simplicity we assume that the excitation field is linearly polarized in the $\hat{\mathbf{z}}$ direction and begin by examining only the $\beta_{z z z}$ component of this hyperpolarizability, denoted $\beta$. If the excitation light has frequency $\omega$, the induced dipole moment at frequency $2 \omega$ will be given by

$$
\boldsymbol{\mu}_{2 \omega}=1 / 2 \beta E_{\omega}^{2} \hat{\mathbf{z}},
$$

where $E_{\omega}$ is the excitation field amplitude and we have used the Taylor convention. ${ }^{19}$ The radiated secondharmonic far field at an inclination $\psi$ from the $z$ axis is

$$
\mathbf{E}_{2 \omega}(\psi)=-\frac{\mu_{2 \omega} \omega^{2}}{\pi \varepsilon_{0} c^{2} r} \sin (\psi) \exp (-2 i \omega[t]) \hat{\boldsymbol{\psi}},
$$

where $\varepsilon_{0}$ is the free-space permittivity, $c$ is the speed of light, $r$ is the observation distance from the dipole, and $[t]$ is the corresponding retarded time. The resultant power per differential solid angle at an inclination $\psi$, in units of photons/second, may be expressed as

$$
P_{2 \omega}(\psi)=\frac{3}{16 \pi} \sigma_{\mathrm{SHG}} \sin ^{2}(\psi) I_{\omega}^{2},
$$

where $I_{\omega}$ is the excitation intensity in units of (photons/ second)/area and

$$
\sigma_{\mathrm{SHG}}=\frac{4 n_{2 \omega} \hbar \omega^{5}}{3 \pi n_{\omega}^{2} \varepsilon_{0}^{3} c^{5}}|\beta|^{2}
$$

( $n_{\omega}$ and $n_{2 \omega}$ are the indices of refraction at $\omega$ and $2 \omega$; $\hbar$ is Planck's constant). We have defined $\sigma_{\mathrm{SHG}}$ such that the total SHG power, obtained by integration of Eq. (3) over all solid angles, reduces to the simple expression

$$
P_{\mathrm{SHG}}=1 / 2 \sigma_{\mathrm{SHG}} I_{\omega}^{2}
$$

(the extra factor of $1 / 2$ stems from our description of powers in units of photons/second rather than in watts).

We recall that the fluorescence power emitted by a dipole undergoing two-photon excitation can be expressed similarly as

$$
P_{\mathrm{TPEF}}=1 / 2 \sigma_{\mathrm{TPEF}} I_{\omega}^{2},
$$

where $\sigma_{\mathrm{TPEF}}$ is the two-photon fluorescence (or action) cross section, defined by the two-photon absorption cross section multiplied by the fluorescence quantum yield. ${ }^{20}$ In view of the similarity between Eqs. (5) and (6), we may regard $\sigma_{\mathrm{SHG}}$ as the cross section for SHG of an individual dipole. In particular, $\sigma_{\mathrm{TPEF}}$ and $\sigma_{\mathrm{SHG}}$ may be expressed in the same units for direct comparison. We note here that $\sigma_{\mathrm{SHG}}$ depends on the square of the magnitude of the molecular first hyperpolarizability, whereas $\sigma_{\mathrm{TPEF}}$ depends on the imaginary part of the second hyperpolarizability. ${ }^{21}$ As such, $\sigma_{\mathrm{SHG}}$ tends to be much smaller than $\sigma_{\mathrm{TPEF}}$ in practice.

\section{B. Coherent Summation}

We now turn to the case of a collection of molecules driven by a field $\mathbf{E}_{\omega}$ and generalize the procedure described above to evaluate the resultant SHG radiation angular distribution and power. We restrict ourselves to the geometry shown in Fig. 1, in which the dipoles are assumed to be uniformly distributed in a two-dimensional $x-y$ plane (membrane plane) and illuminated by a highly focused excitation beam propagating side-on in the $\hat{\mathbf{x}}$ direction and polarized in the $y-z$ plane (the fact that we use a side-on geometry rather than a head-on geometry will become evident). Our basic strategy consists in coarsegrain averaging the molecular dipole moments over membrane surface areas whose dimensions are small compared with the radiation wavelength but large enough to encompass large numbers of molecules. In this way we may define a local induced macroscopic dipole moment per unit molecular surface density:

$$
\mu_{2 \omega, i}(x, y)=1 / 2 E_{\omega}^{2}(x, y) \sum_{j, k}\left\langle\beta_{i j k}\right\rangle \hat{\varepsilon}_{j} \hat{\varepsilon}_{k},
$$

where $E_{\omega}(x, y)$ is the complex amplitude of the driving field at the position $(x, y)$ on the membrane, $\hat{\boldsymbol{\epsilon}}$ is its polarization direction, $\langle\beta\rangle$ symbolizes a local ensemble average of the molecular hyperpolarizabilities (we shall describe 


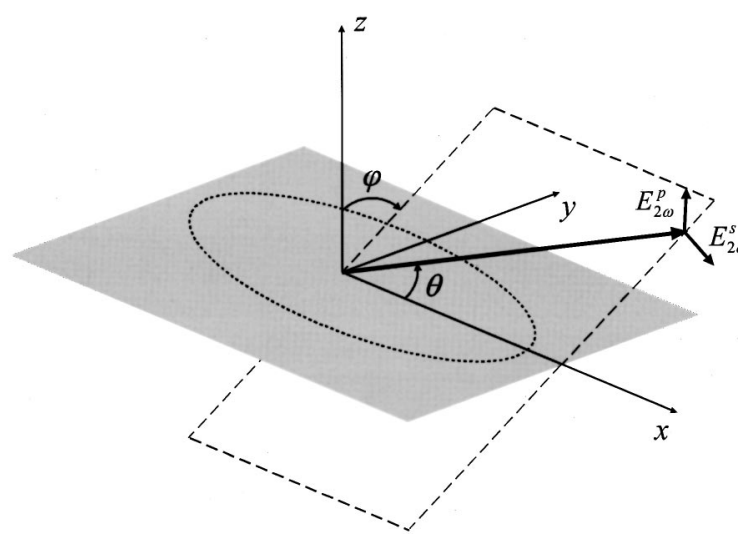

Fig. 1. Coordinate system defining the SHG emission direction. The membrane surface (shaded; $z=0$ ) is approximated to be planar at the length scales considered. The focused excitation beam propagates in the $+x$ direction. The resultant SHG is mostly confined to an interaction area schematically depicted as a dotted ellipsoid and is radiated in the directions defined by $\theta$ and $\varphi$ (thick arrow), with polarization components parallel to $\left(E_{2 \omega}^{p}\right)$ and perpendicular to $\left(E_{2 \omega}^{s}\right)$ the emission plane (shown dashed).

this average in more detail below), and we have neglected local field effects. We emphasize that our term "macroscopic" here is still microscopic relative to wavelength scales. Thus all the molecules taken in the coarse-grain average at a given position $(x, y)$ are driven in phase with one another, and the net local dipole moment per unit area generated at this position is simply $N_{s} \boldsymbol{\mu}_{2 \omega}(x, y)$, where $N_{s}$ is the molecular surface density. The phase of $\boldsymbol{\mu}_{2 \omega}(x, y)$ at different positions is determined by the phase of the driving field $\mathbf{E}_{\omega}(x, y)$. To calculate the radiation generated by a global membrane surface, we then treat the local dipole moments at each position on the membrane plane as elemental radiators and coherently sum their radiated electric fields while taking their relative phases and amplitudes into account.

As we emphasized in Section 1, the excitation field cannot be regarded as a simple collimated beam here, and indeed a full calculation that takes diffraction and polarization into account shows that the field's amplitude and phase cannot even be expressed analytically in the case of tight focusing. ${ }^{22}$ It has been shown, however, that as far as nonlinear interactions are concerned one may closely approximate a tightly focused field by assuming that its amplitude is Gaussian in both the axial and the lateral directions about the focal center and that its phase near the focal center progresses linearly. That is, we can write

$$
E_{\omega}(x, y)=-i E_{\omega} \exp \left(-\frac{x^{2}}{w_{x}^{2}}-\frac{y^{2}}{w_{y}^{2}}+i \xi k_{\omega} x\right),
$$

where $w_{x}$ and $w_{y}$ are the axial and the lateral field waists, respectively (explicit expressions for these may be found in App. B of Ref. 23), $k_{\omega}=n_{\omega} \omega / c$ is the local wave vector, and $\xi$ is a parameter that characterizes the phase shift experienced by a Gaussian beam in the vicinity of a focal center. This phase shift is commonly referred to as a Gouy shift or a phase anomaly. ${ }^{22}$ In the case of weak focusing, $\xi$ may be approximated by $\left(1-2 / k_{\omega}^{2} w_{y}^{2}\right)$, whereas for tight focusing this expression tends to be a slight overestimate (see Appendix A). We emphasize again that the field in Eq. (8) differs from a collimated beam in two respects: It experiences a local axial amplitude variation about the focal center as well as a phase shift. Both of these effects play a considerable role in the structure of the resultant SHG.

By adopting the coordinate system illustrated in Fig. 1, we proceed to calculate the second-harmonic far field radiated in the direction $(\theta, \varphi)$. We begin by writing the contribution to the radiated electric field from the local induced polarization per unit molecular surface density and at the focal center only [indicated by the superscript ${ }^{(0)}$; $\left.\boldsymbol{\mu}_{2 \omega}^{(0)}=\boldsymbol{\mu}_{2 \omega}(0,0)\right]$. This is given by

$$
\mathbf{E}_{2 \omega}^{(0)}(\theta, \varphi)=\left(\begin{array}{c}
E_{2 \omega}^{(0) p}(\theta, \varphi) \\
E_{2 \omega}^{(0) s}(\theta, \varphi)
\end{array}\right)=\frac{\eta}{r} \mathbf{M} \cdot \boldsymbol{\mu}_{2 \omega}^{(0)},
$$

where $\eta=\omega^{2} / \pi \varepsilon_{0} c^{2}$ and $\mathbf{M}$ is the projection matrix, defined by

$$
\mathbf{M}\left[\begin{array}{c}
\hat{\boldsymbol{\theta}} \\
\hat{\boldsymbol{\varphi}}
\end{array}\right]=\left[\begin{array}{ccc}
-\sin \theta & \cos \theta \sin \varphi & \cos \theta \cos \varphi \\
0 & \cos \varphi & -\sin \varphi
\end{array}\right]
$$

That is, $E_{2 \omega}^{p}$ is the amplitude of $\mathbf{E}_{2 \omega}$ in the $\hat{\boldsymbol{\theta}}$ direction and $E_{2 \omega}^{s}$ is the amplitude of $\mathbf{E}_{2 \omega}$ in the $\hat{\boldsymbol{\varphi}}$ direction. The total radiated field in the propagation direction $(\theta, \varphi)$ is then given by the coherent summation of the contributions from the local induced polarization at all points $x$ and $y$, with their associated spatially dependent phase shifts taken into account. We find then that

$$
\begin{aligned}
& \mathbf{E}_{2 \omega}(\theta, \varphi) \\
& =\frac{\eta N_{s}}{r} \iint \mathbf{M} \cdot \boldsymbol{\mu}_{2 \omega}(x, y) \\
& \quad \times \exp \left[-i k_{2 \omega}(x \cos \theta+y \sin \theta \sin \varphi)\right] \mathrm{d} x \mathrm{~d} y,
\end{aligned}
$$

where $k_{2 \omega}=2 n_{2 \omega} \omega / c$. On integration, Eq. (11) yields

$$
\mathbf{E}_{2 \omega}(\theta, \varphi)=N A(\theta, \varphi) \mathbf{E}_{2 \omega}^{(0)}(\theta, \varphi),
$$

where we have introduced the parameters

$$
\begin{aligned}
N & =\frac{\pi}{2} w_{x} w_{y} N_{s}, \\
A(\theta, \varphi) & =\exp \left\{-\frac{k_{2 \omega}^{2}}{8}\left[w_{x}^{2}\left(\cos \theta-\xi^{\prime}\right)^{2}+w_{y}^{2}(\sin \theta \sin \varphi)^{2}\right]\right\}
\end{aligned}
$$

and $\xi^{\prime}=\xi n_{\omega} / n_{2 \omega}$. The physical meanings of $N$ and $A(\theta, \varphi)$ are as follows: $N$ defines the effective total number of molecules that contribute to the generation of second-harmonic light. This definition is identical to that detailed in Ref. 23 for two-photon excitation, though here it applies to a two-dimensional geometry. Accordingly, the effective total surface area that produces second-harmonic light is simply $N / N_{s}$. Because the molecules involved in SHG are spatially distributed on this surface area, the resultant angular profile of the SHG radiation is much more complicated in structure than that of a simple elemental radiator. In particular, we observe from Eq. (12) that the emission pattern of the SHG is the product of two terms: The first is the pattern that arises from an elemental dipole radiator $\mathbf{E}_{2 \omega}^{(0)}(\theta, \varphi)$ at the focal 
center, and the second is an angular modulation term defined by the scalar function $A(\theta, \varphi)$. As we shall see below, the effect of the latter modulation term on the radiation structure is quite dramatic.

Finally, the radiated second-harmonic powers in the $p$ and $s$ polarization and in the solid angle defined by $(\theta, \varphi)$ are given by

$$
P_{2 \omega}^{p, s}(\theta, \varphi)=1 / 2 n_{2 \omega} \varepsilon_{0} c r^{2}\left|E_{2 \omega}^{p, s}(\theta, \varphi)\right|^{2},
$$

and the total radiated power obtained by integration over all angles becomes

$$
\begin{aligned}
P_{\mathrm{SHG}}= & P_{\mathrm{SHG}}^{p}+P_{\mathrm{SHG}}^{s} \\
= & \frac{4 \pi}{3} n_{2 \omega} \varepsilon_{0} c \eta^{2} N^{2} \\
& \times\left[\Theta_{x} \mu_{2 \omega, x}^{(0) 2}+\Theta_{y} \mu_{2 \omega, y}^{(0) 2}+\Theta_{z} \mu_{2 \omega, z}^{(0) 2}\right],
\end{aligned}
$$

where we have introduced the angular structure parameters

$$
\begin{aligned}
& \Theta_{x}=\frac{3}{8 \pi} \int A^{2}(\theta, \varphi) \sin ^{3} \theta \mathrm{d} \theta \mathrm{d} \varphi, \\
& \Theta_{y}=\frac{3}{8 \pi} \int A^{2}(\theta, \varphi)\left[1-\sin ^{2} \theta \sin ^{2} \varphi\right] \sin \theta \mathrm{d} \theta \mathrm{d} \varphi, \\
& \Theta_{z}=\frac{3}{8 \pi} \int A^{2}(\theta, \varphi)\left[1-\sin ^{2} \theta \cos ^{2} \varphi\right] \sin \theta \mathrm{d} \theta \mathrm{d} \varphi .
\end{aligned}
$$

The contributions to the total power from the various directional components of the induced second-harmonic dipoles are therefore readily identified. We note that if $A(\theta, \varphi)=1$, as is the case for a single elemental dipole radiator, then $\Theta_{x}=\Theta_{y}=\Theta_{z}=1$.

\section{Membrane Hyperpolarizability}

In the derivation of Eqs. (15) and (16), no assumptions have been made about the dipole vector $\boldsymbol{\mu}_{2 \omega}^{(0)}$, which may be taken as arbitrary. For our considerations, however, we shall assume that $\boldsymbol{\mu}_{2 \omega}^{(0)}$ is generated by molecules that are quasi-uniaxial. That is, if we define a coordinate system $(X, Y, Z)$ that is proper to a molecule, then the molecular first hyperpolarizability is dominated largely by the single component $\beta_{z z z}$. Moreover, we assume that the molecules are preferentially aligned in the lipid membrane such that, on average, the molecular $Z$ axis is in the same direction as the $z$ axis defined by the membrane, though we do not otherwise specify the distribution that governs tilt angles $\alpha$.

The molecular $X$ and $Y$ axes, in turn, are assumed to be uniformly randomly oriented. In particular, we discount any possible correlation between the $X$ and $Y$ orientations and tilt angle $\alpha$. With the above assumptions in mind, we may derive a coarse-grained local ensemble average for the hyperpolarizability that is valid over regions whose dimensions are small compared with the optical wavelength but large enough to encompass a large number of molecules. Expressed in the membrane coordinate system $(x, y, z)$, the nonzero components of this first hyperpolarizability are given by

$$
\begin{aligned}
& \beta_{x x z}=\beta_{x z x}=\beta_{y y z}=\beta_{y z y} \equiv \beta_{+}, \\
& \beta_{x y z}=\beta_{x z y}=-\beta_{y x z}=-\beta_{y z x} \equiv \beta_{-}, \\
& \beta_{z x x}=\beta_{z y y} \equiv \beta_{t}, \\
& \beta_{z z z} \equiv \beta_{z},
\end{aligned}
$$

where we have used the definitions

$$
\begin{aligned}
& \beta_{+}=\left\langle\cos ^{3} \alpha\right\rangle B_{+}+1 / 2\left\langle\sin ^{2} \alpha \cos \alpha\right\rangle\left(B_{Z}-B_{T}\right) \\
& \beta_{-}=1 / 2\left\langle 3 \cos ^{2} \alpha-1\right\rangle B_{-} \\
& \beta_{t}=\left\langle\cos ^{3} \alpha\right\rangle B_{T}+1 / 2\left\langle\sin ^{2} \alpha \cos \alpha\right\rangle\left(B_{Z}+B_{T}-2 B_{+}\right), \\
& \beta_{z}=\left\langle\cos ^{3} \alpha\right\rangle B_{Z}+\left\langle\sin ^{2} \alpha \cos \alpha\right\rangle\left(B_{T}+2 B_{+}\right)
\end{aligned}
$$

where \langle\rangle signifies an ensemble average and

$$
\begin{aligned}
& B_{+}=1 / 2\left(\beta_{X X Z}+\beta_{Y Y Z}\right), \\
& B_{-}=1 / 2\left(\beta_{X Y Z}-\beta_{Y X Z}\right), \\
& B_{T}=1 / 2\left(\beta_{Z X X}+\beta_{Z Y Y}\right), \\
& B_{Z}=\beta_{Z Z Z} .
\end{aligned}
$$

Having derived the effective molecular hyperpolarizability in the membrane frame, we may now derive the induced second-harmonic dipole at the focal center $\boldsymbol{\mu}_{2 \omega}^{(0)}$ from Eq. (7). We consider the case when the driving field is polarized in the $y-z$ plane and write $\hat{\boldsymbol{\varepsilon}}$ $=(0, \sin \phi, \cos \phi)$, where $\phi$ represents the inclination of the polarization direction from the $z$ axis. A straightforward application of Eqs. (7) and (18) then yields

$\boldsymbol{\mu}_{2 \omega}^{(0)}=1 / 2 E_{\omega}^{2}\left(\beta_{-} \sin 2 \phi, \beta_{+} \sin 2 \phi, \beta_{z} \cos ^{2} \phi+\beta_{t} \sin ^{2} \phi\right)$.

Finally, by inserting Eq. (21) into Eqs. (9), (12), and (15), we obtain the $p$ and $s$ polarization components of the radiated second-harmonic power.

\section{Uniaxial Hyperpolarizability}

To distill the basic features of our results, we restrict ourselves here to the simplifying case in which the molecules in the membrane are perfectly oriented in the $\hat{z}$ direction (i.e., $\alpha=0$ ) and strictly uniaxial, such that the only nonzero component to the molecular first hyperpolarizability is $\beta_{Z Z Z}$ (i.e., $\beta_{+}=\beta_{-}=\beta_{t}=0 ; \beta_{z}=\beta_{Z Z Z}$ ). We also begin by considering the case in which the driving field is polarized in the $\hat{z}$ direction (i.e., $\phi=0$ ). This is the same case as examined in subsection 2.A for a single molecule, with the only difference being that here we consider the effects of a planar distribution of molecules. As before, we find at the focal center

$$
\boldsymbol{\mu}_{2 \omega}^{(0)}=1 / 2 \beta_{z} E_{\omega}^{2} \hat{\mathbf{z}}
$$

which again is the induced dipole moment per unit molecular surface density. The radiated power from the distribution of induced dipole moments about the focal center, expressed in photons/second, is then given by 


$$
\begin{aligned}
& P_{2 \omega}^{p}(\theta, \varphi)=\frac{3}{16 \pi} \sigma_{\mathrm{SHG}} N^{2} A^{2}(\theta, \varphi) \cos ^{2} \theta \cos ^{2} \varphi I_{\omega}^{2}, \\
& P_{2 \omega}^{s}(\theta, \varphi)=\frac{3}{16 \pi} \sigma_{\mathrm{SHG}} N^{2} A^{2}(\theta, \varphi) \sin ^{2} \varphi I_{\omega}^{2},
\end{aligned}
$$

and the integrated total power is

$$
P_{\mathrm{SHG}}=1 / 2 \Theta_{z} N^{2} \sigma_{\mathrm{SHG}} I_{\omega}^{2} .
$$

For a single radiating dipole, $\Theta_{z}=1$; however, for a distribution of many dipoles, $\Theta_{z}<1$ in general. The angular pattern of the radiated SHG light is governed mainly by the function $A(\theta, \varphi)$. The structure of $A(\theta, \varphi)$ critically depends on the interaction area and on $\xi^{\prime}$ and exhibits two symmetric peaks, at $\theta_{\text {peak }}= \pm \cos ^{-1}\left(\xi^{\prime}\right)$ (Fig. 2). This bidirectional nature of the SHG stems from the fact that the excitation light is subject to an effective increase in wavelength owing to the phase anomaly near the focal center. As a result, the $\mathrm{SHG}$ radiation must be emitted at an angle $\pm \theta_{\text {peak }}$ relative to the excitation propagation direction to be properly phase matched. We assume throughout this paper that $\xi$ is roughly constant within the SHG excitation area. This approximation is justified in Appendix A. We emphasize here that $\xi$ is intimately linked to the interaction area and may not be regarded as an independent parameter. When the interaction area is reduced, then so too is $\xi$, which leads to a widening of the angular separation between the two lobes as well as a broadening of the lobes themselves. In particular, a reduction in the axial and lateral waists of the excitation beam leads to a broadening of the lobe profiles along the $\theta$ and $\varphi$ directions, roughly respectively.

A direct comparison can be made between the total powers emitted in the cases of SHG and TPEF. Noting that the total TPEF power scales with $N$, we find that

$$
\frac{P_{\mathrm{SHG}}}{P_{\mathrm{TPEF}}}=2 \sqrt{2} \Theta_{z} N \frac{\sigma_{\mathrm{SHG}}}{\sigma_{\mathrm{TPEF}}},
$$

where the factor $2 \sqrt{2}$ stems from the volume contrast of a three-dimensionally Gaussian fluorescence excitation volume. $^{23}$

Because the SHG radiation is emitted into two well-defined lobes, small-angle approximations about $\theta \approx \pm \theta_{\text {peak }}$ and $\varphi \approx 0$ may be used to provide an estimate

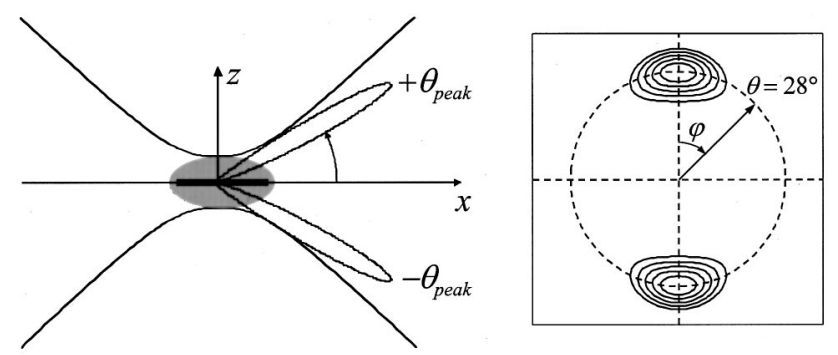

Fig. 2. Left, an excitation beam propagating in the $x$ direction and polarized along the $z$ axis is focused (side-on) onto the membrane of a labeled lipid vesicle. Only a small surface area (thick segment; side view) of this much larger vesicle contributes to SHG. Phase matching between the SHG and excitation fields causes the SHG radiation to be double peaked in the forward direction. Right, far-field power distribution of the SHG radiation. of the structure parameter $\Theta_{z}$. A straightforward evaluation of Eqs. (17) with these approximations yields

$$
\Theta_{z} \approx \frac{3 \xi^{\prime 2}}{k_{2 \omega}^{2} w_{x} w_{y} \sqrt{1-\xi^{\prime 2}}}
$$

Bearing in mind the approximate dependence of the focal area on the numerical aperture (NA) of the excitation beam $\left(w_{x} \sim \mathrm{NA}^{-2} ; w_{y} \sim \mathrm{NA}^{-1}\right)$, we can infer the following relations: $\quad N \sim \mathrm{NA}^{-3}, I_{\omega} \sim \mathrm{NA}^{2}$, and for low NA we can approximate $\Theta_{z} \sim \mathrm{NA}^{2}$ (see Appendix A). We find then that the total emitted SHG power is roughly independent of NA for NA $<0.8$. For higher NA's, the SHG power diminishes. In comparison, the total emitted TPEF power scales as NA in a two-dimensional geometry.

\section{E. Polarization Anisotropy}

In our discussions so far we have assumed that the membrane geometry was planar. In our experiments, however, the membranes are in fact spherical, with diameters typically in the $20-50-\mu \mathrm{m}$ range. The excitation beam waist at the focal center is typically of submicrometer size; hence the assumption that the membrane is planar over this dimension is entirely valid. We note, however, that the membrane coordinate frame as defined above is not the same as the laboratory coordinate frame. In particular, if the excitation beam polarization is linear and fixed in the laboratory frame, it will appear to tilt in the membrane frame, depending on which portion of the membrane surface is illuminated. If we scan only along an equatorial cross section of the membrane, the polarization direction will rotate relative to the membrane's $z$ axis with an angle $\phi$, which spans 0 to $2 \pi$. In the case of perfectly aligned uniaxial molecules $\left(\beta_{+}=\beta_{-}=\beta_{t}=0\right)$, the induced local dipole moment for an angle $\phi$ as obtained from Eq. (21) is simply

$$
\boldsymbol{\mu}_{2 \omega}^{(0)}=1 / 2 E_{\omega}^{2}\left(0,0, \beta_{z} \cos ^{2} \phi\right) .
$$

The resultant $p$ and $s$ polarizations of the radiated field in the local membrane coordinate frame are then obtained from Eq. (9), and the total radiation power generated locally along the membrane equator is found to vary as $\cos ^{4} \phi$.

Experimentally it is difficult to isolate the $p$ and $s$ components of the radiated field because these are not fixed in the laboratory frame. We can transform them, however, into orthogonal polarization components, which are fixed in the laboratory frame through the relation

$$
\left(\begin{array}{l}
E_{2 \omega}^{\|} \\
E_{2 \omega}^{\perp}
\end{array}\right)=\left[\begin{array}{cc}
\cos (\varphi-\phi) & -\sin (\varphi-\phi) \\
\sin (\varphi-\phi) & \cos (\varphi-\phi)
\end{array}\right]\left(\begin{array}{l}
E_{2 \omega}^{p} \\
E_{2 \omega}^{s}
\end{array}\right) .
$$

Here $E_{2 \omega}^{\|}$and $E_{2 \omega}^{\perp}$ represent the radiated electric field components parallel and perpendicular, respectively, to the excitation beam's polarization direction. These latter polarization components can be isolated quite simply, as is shown below.

Following steps similar to those used in deriving Eq. (16), we can derive the total radiated SHG powers along the $\|$ and $\perp$ polarizations. These are given by 


$$
\begin{aligned}
& P_{\mathrm{SHG}}^{\|}=\frac{4 \pi}{3} n_{2 \omega} \varepsilon_{0} c \eta^{2} N^{2} \mu_{2 \omega, z}^{(0) 2}\left[\left(\Theta_{z}-2 \Theta_{z}^{\prime}\right) \cos ^{2} \phi+\Theta_{z}^{\prime}\right], \\
& P_{\mathrm{SHG}}^{\perp}=\frac{4 \pi}{3} n_{2 \omega} \varepsilon_{0} c \eta^{2} N^{2} \mu_{2 \omega, z}^{(0) 2}\left[\left(\Theta_{z}-2 \Theta_{z}^{\prime}\right) \sin ^{2} \phi+\Theta_{z}^{\prime}\right],
\end{aligned}
$$

where we have introduced the auxiliary angular structure parameter

$$
\begin{aligned}
\Theta_{z}^{\prime}= & \frac{3}{8 \pi} \int A^{2}(\theta, \varphi) \\
& \times\left[(1-\cos \theta)^{2} \sin ^{2} \varphi \cos ^{2} \varphi\right] \sin \theta \mathrm{d} \theta \mathrm{d} \varphi .
\end{aligned}
$$

Although the above relations are exact, in most cases of interest $\Theta_{z}^{\prime}$ is considerably smaller than $\Theta_{z}$ and can safely be neglected. We then find that the powers emitted into the orthogonal polarization components $P_{\mathrm{SHG}}^{\|}$and $P_{\mathrm{SHG}}^{\perp}$ vary, respectively, as $\cos ^{6} \phi$ and $\cos ^{4} \phi \sin ^{2} \phi$ along the membrane equator. We emphasize that the above results apply to rigorously uniaxial and well-oriented molecules only. In practice, not all molecules have these attributes, as will be seen below.

\section{SHG CROSS SECTION}

The molecule used in our experimental investigation is the lipophilic styryl dye $N$-(4-sulfobutyl)-4-(4-(dihexylamino)styryl)pyridinium (Di-6-ASPBS). The donor-( $\pi$ bridge)-acceptor structure of this molecule permits significant charge transfer along its major $Z$ axis, resulting in a large first hyperpolarizability component $\beta_{Z Z Z} \cdot{ }^{24-26}$ Donor-( $\pi$-bridge $)$-acceptor molecules have been found to be well described by a two-state model. ${ }^{15,27}$ When driven near resonance, $\beta_{Z Z Z}$ may be approximated by

$$
\begin{aligned}
\beta_{Z Z Z}(-2 \omega, \omega, \omega) & \\
= & \frac{2}{\hbar^{2}} \mu_{e g}^{2} \Delta \mu\left[\frac{1}{\left(\omega_{e g}-2 \omega+i \Gamma\right)\left(\omega_{e g}-\omega+i \Gamma\right)}\right. \\
& +\frac{1}{\left(\omega_{e g}-\omega+i \Gamma\right)\left(\omega_{e g}+\omega-i \Gamma\right)} \\
& \left.+\frac{1}{\left(\omega_{e g}+2 \omega-i \Gamma\right)\left(\omega_{e g}+\omega-i \Gamma\right)}\right],
\end{aligned}
$$

where $\omega_{e g}$ and $\mu_{e g}$ are, respectively, the transition frequency and the dipole transition moment, $\Delta \mu$ is the difference between excited-state and ground-state dipole moments and $\Gamma$ is a phenomenological damping constant. Although no direct experimental measurements of $\beta_{Z Z Z}$ in membrane are available, the absorption spectrum of Di-6-ASPBS in membrane allows us to infer a charge transfer energy $\hbar \omega_{\text {eg }}=2.56 \mathrm{eV}$, a transition moment $\mu_{e g} \approx 10 \mathrm{D}$, and a damping factor $\Gamma \approx 0.19 \mathrm{eV}$, all specific to a membrane environment. In addition, previous electrochromism measurements of similar dyes in membrane $^{28}$ allow us to estimate that $\Delta \mu \approx 16 \mathrm{D}$ for Di-6-ASPBS. We may therefore predict a large static first hyperpolarizability of roughly $\beta(0) \approx 2$ $\times 10^{-48} \mathrm{C} \mathrm{m}^{3} \mathrm{~V}^{-2}$. This value is enhanced when the molecule is excited near resonance. In particular, for an excitation wavelength of $880 \mathrm{~nm}$, we find that $\beta \approx 1$ $\times 10^{-47} \mathrm{C} \mathrm{m}^{3} \mathrm{~V}^{-2}, \quad$ or, equivalently, $\sigma_{\mathrm{SHG}} \approx 1$ $\times 10^{-3} \mathrm{GM}\left(1 \mathrm{GM}=10^{-50} \mathrm{~cm}^{4} /\right.$ photon $\left.\mathrm{s}^{-1}\right)$. For comparison, the TPEF cross section of Di-6-ASPBS in membrane at the same excitation wavelength has been estimated to be $\sigma_{\mathrm{TPEF}} \approx 30 \mathrm{GM}$, based on TPEF measurements in ethanol. We note that, despite the fact that $\sigma_{\mathrm{SHG}}$ is $\sim 4$ orders of magnitude smaller than $\sigma_{\mathrm{TPEF}}$ for a single molecule, we may benefit from the fact that SHG field amplitudes add coherently in the case of a large number of molecules, as shown in Eq. (26). In practice and for standard dye labeling densities, we can easily obtain SHG and TPEF powers that are comparable, as we show below.

\section{EXPERIMENTAL ANALYSIS}

\section{A. SHG and TPEF Imaging of Membranes}

Our experimental apparatus is a home-built scanning SHG TPEF microscope, the basics of which are shown in Fig. 3. The excitation source is a mode-locked Ti:sapphire laser (Spectra-Physics), which delivers $\sim 80$-fs pulses at an $81-\mathrm{MHz}$ repetition rate. The laser light is focused into the sample with a water-immersion microscope objective (Olympus, LUMPlanFL60 $\times$ W/IR) and the resultant SHG is collected in the forward direction, while the TPEF is collected in the backward direction. Our sample consists of giant unilamellar vesicles (GUV's) made from a pure lipid in water. These serve as model systems for the study of SHG radiation from membrane surfaces. The lipid type is 1,2-dioleoyl-sn-glycero-3phosphocholine (Avanti, DOPC), which is in the fluid phase $L_{\alpha}$ at room temperature. The vesicles are labeled at 1 mol. \% with Di-6-ASPBS; preparation and labeling are described elsewhere. ${ }^{29}$ Calcium ions are added to promote adhesion between adjacent vesicles, and the glass slide is coated with poly- $L$-lysine (Sigma P8920) to prevent sticking and bursting.

Figure 4 shows SHG and TPEF images of three GUV's of nearly equal radii that have adhered to form a foam. The GUV's are labeled with Di-6-ASPBS and acquired under the conditions described above. SHG scales with the

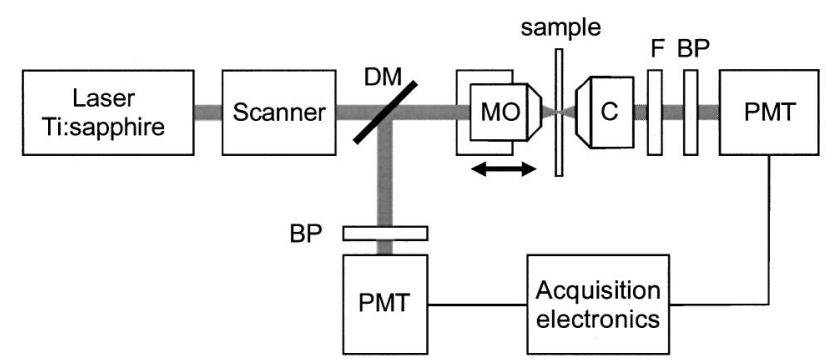

Fig. 3. Experimental layout: a Ti:sapphire laser beam is focused into a sample with a microscope objective (MO). The transmitted SHG is collected with a condenser (C), bandpass (BP) filtered, and detected with a photomultiplier tube (PMT). The transmitted laser light is blocked with a colored glass filter (F). The TPEF from the sample is epicollected, discriminated with a dichroic mirror (DM), bandpass (BP) filtered, and detected with a PMT. Three-dimensional images are formed by scanning the laser focal spot in the lateral directions with galvanometermounted mirrors, and in the axial direction by translating the MO. 

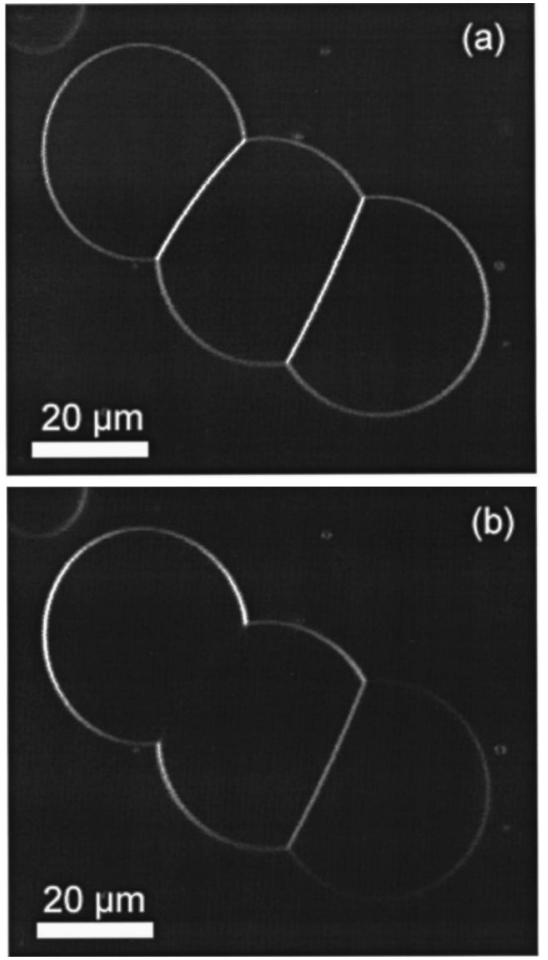

Fig. 4. Simultaneous (a) TPEF and (b) SHG images of three vesicles labeled with Di-6-ASPBS that have adhered to form a foam. The radiating Di-6-ASPBS molecules are more-or-less symmetrically distributed in the adherence regions between the vesicles. In the left region, a symmetric distribution results in a nearly perfect cancellation of SHG. In the right region, the cancellation is imperfect because of a disparity in labeling density. As opposed to SHG radiation, TPEF is independent of molecular distribution because it is incoherent.

square of the excitation intensity and hence leads to the same intrinsic three-dimensional resolution as TPEF, here $510 \mathrm{~nm}$ lateral and $1.9 \mu \mathrm{m}$ axial. An excitation power of less than $1 \mathrm{~mW}$ at the sample provides approximately equal measured powers for both SHG and TPEF, allowing the images to be acquired simultaneously with integration times of $\sim 10 \mu \mathrm{s} /$ pixel. The measured radiated powers are in good agreement with those predicted by the model developed in Section 3. In particular, if we estimate our dye surface density to be $N_{s}=1.5$ $\times 10^{16} \mathrm{~m}^{-2}$, and take into account the fact that essentially all the SHG light is collected because it is highly directional whereas only $\sim 20 \%$ of the radiated fluorescence is epicollected, we find that $P_{\mathrm{SHG}} / P_{\mathrm{TPEF}} \approx 0.5$. To confirm that the light collected in the forward direction is indeed SHG in origin, we routed both the forward and the backward signals into a spectrograph. The SHG spectrum revealed a sharp peak at $440 \mathrm{~nm}$, whereas the TPEF spectrum was broadly distributed about $580 \mathrm{~nm} .{ }^{18}$ The leftmost two vesicles in Fig. 4(a) have nearly equal TPEF brightness; the third vesicle is less bright, presumably owing to reduced labeling. Because SHG scales with the square of the label surface density, the contrast between the two bright vesicles and the third, dimmer, vesicle is enhanced in Fig. 4(b). We also clearly illustrate here the coherent nature of SHG radiation, as opposed to the incoherent nature of TPEF. In regions where the dipole distributions are symmetric, such as in the adhesion zones between the adjacent vesicles, the SHG vanishes, whereas the TPEF does not. We note that the cancellation of SHG is almost perfect in the left zone. It is less perfect in the right zone owing to the disparity in the surface labeling density.

\section{B. SHG Radiation Pattern}

To characterize the angular pattern of the SHG radiation we removed the condenser from our collection optics and replaced it with an oil-immersion microscope objective (Olympus, UPlanFL60×1.25 Oil Iris). We then imaged the back aperture of this objective onto a CCD camera (Cohu 4912/Scion Corporation LG-3 frame grabber). This allowed us to visualize directly the emission angle $\theta$. Figure 5 illustrates the SHG radiation pattern obtained when the laser scanning was restricted to a small patch of membrane on the equatorial slice of a vesicle. The average power at the focus was maintained below $5 \mathrm{~mW}$, permitting a CCD integration time of $\sim 5 \mathrm{~ms} /$ pixel. The two lobes that occur at $\pm \theta_{\text {peak }}$ are manifest. These are the emission directions that correspond to phase matching of the SHG and excitation fields, as predicted by theory. As shown, however, the two lobes are not perfectly cylindrically symmetric about $\theta=0$. In particular, the left lobe curves outward rather than inward. This may be the result of the local membrane curvature, which is not taken into account in our theory. In particular, when the membrane curvature was reversed, the lobe symmetry also became reversed. We also point out that there was a slight index-of-refraction mismatch between the insides and the outsides of our vesicles. As a result, the lobe that is directed toward the inside of a vesicle experiences a slight distortion owing to the lensing effect when it exits the vesicle, which may further contribute to the observed asymmetry in the lobes.

When the laser was allowed to scan over an entire vesicle cross section, the angular radiation pattern became annular, as shown in Fig. 6. The radiation pattern was observed to be highly sensitive to the inclination of the local membrane plane relative to the propagation axis. For example, in images generated from cross sec-

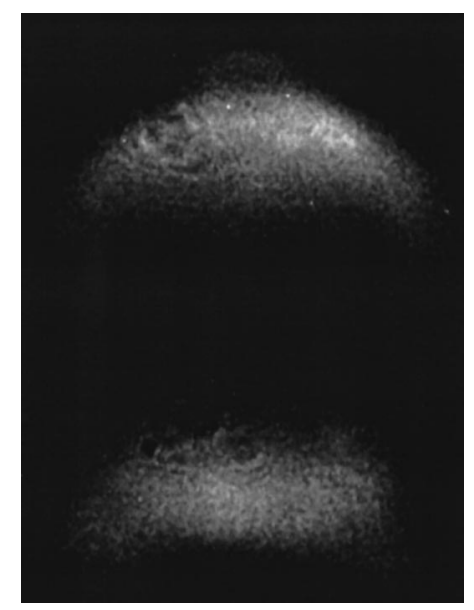

Fig. 5. CCD image of the back aperture of the SHG collection objective. The excitation beam is scanned only over a small portion of an equatorial slice of a GUV membrane. The doublepeaked angular distribution of the SHG radiation is apparent. 

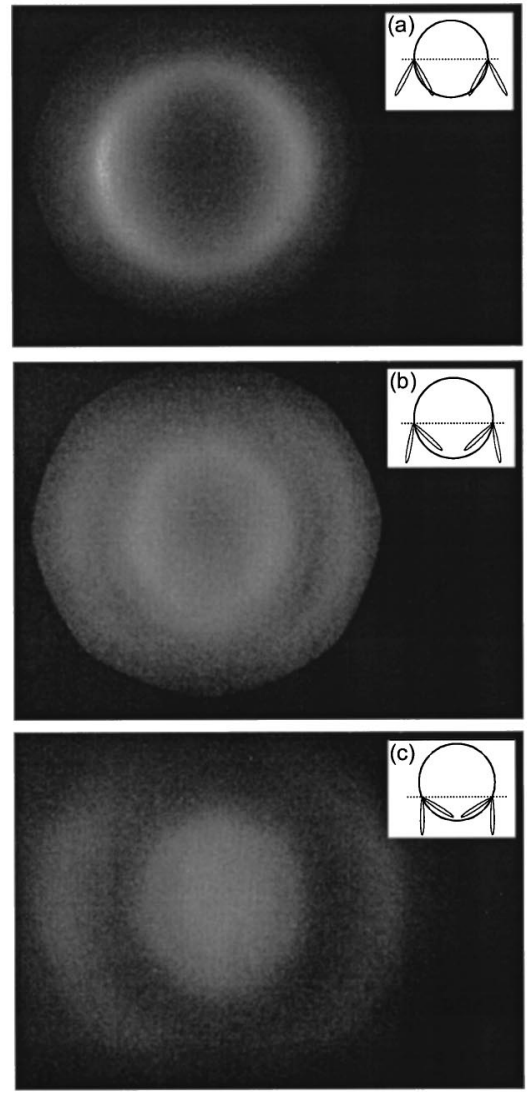

Fig. 6. Stack of CCD images of the back aperture of the SHG collection objective. The excitation beam, incident from above in the insets, is scanned through full cross sections of a GUV at various latitudes. The equatorial cross section is scanned for image (a), leading to a single annular ring at $\theta_{\text {peak }}$, whereas latitudes below the equator are scanned for images (b) and (c). The CCD images were integrated over times long enough to permit multiple scans. From image (a) we deduce that $\theta_{\text {peak }} \approx 24^{\circ}$.

tions below the equatorial plane, two annular rings became apparent. The origin of these rings is made clear in Fig. 6. We note that the radius of the annular ring obtained from the equatorial plane provides a precise determination of $\theta_{\text {peak }}$. In our case, from Fig. 6(a) and correcting for the index mismatch that is caused by the condenser glass, we find experimentally $\theta_{\text {peak }} \approx 24^{\circ}$, corresponding to $\xi^{\prime} \approx 0.91$. This value of $\xi^{\prime}$ is somewhat larger than that predicted from numerical calculations (see Appendix A). A possible reason for this discrepancy is that, as mentioned above, one lobe experiences a slight deviation on transit through the vesicle while the other does not. Furthermore, we assumed in our calculations that the back aperture of the excitation objective was completely and uniformly filled. Such was not the case in practice, meaning that our focal spot was probably somewhat larger than specified.

\section{SHG Polarization Analysis}

To conclude our experimental analysis, we briefly examine some polarization anisotropy properties of the SHG radiation obtained from vesicle membranes. In particular, we consider the case in which the excitation light is linearly polarized and we can select the corresponding parallel and cross-polarized components of the detected
SHG radiation. We perform this selection simply by inserting a linear polarizer between the condenser and the SHG detection photomultiplier tube in Fig. 2. Depending on the orientation of this polarizer relative to the excitation polarization detection, we can effectively isolate the $P_{\mathrm{SHG}}^{\|}$and $P_{\mathrm{SHG}}^{\perp}$ components of the SHG radiation, as described in Subsection 2.E. Our results, for example, for Di-6-ASPBS labeling of GUV's are presented in Fig. 7. We note that we do not fully recover the respective $\cos ^{6} \phi$ and $\cos ^{4} \phi \sin ^{2} \phi$ angular dependencies for $P_{\mathrm{SHG}}^{\|}$and $P_{\mathrm{SHG}}^{\perp}$, as predicted from our simplified model. Though there may be several explanations for this, the most probable is that the assumptions that Di-6-ASPBS is strictly uniaxial and that it is perfectly aligned in the $\hat{z}$ direction when it is inserted into a membrane are overly simplistic. For example, it has been suggested ${ }^{30}$ that a molecule closely related to Di-6-ASPBS may possess a significant transverse polarizability component $B_{T}$ in the molecule frame [and hence $\beta_{t}$ in the membrane frame; see Eqs. (18)-(20)]. Such a transverse component would add a $\sin ^{2} \phi$ component to $\mu_{2 \omega, z}^{(0)}$ and hence would significantly modify the expected angular dependencies of $P_{\mathrm{SHG}}^{\|}$and $P_{\mathrm{SHG}}^{\perp}$, yielding a closer fit to experiment. Moreover, even in the case of a rigorously uniaxial polarizability, any deviations from perfect alignment would further complicate matters by introducing a nonzero $\mu_{2 \omega, y}^{(0)}$ component. Finally, we assumed in Subsection 2.E that the active SHG surface area remained constant everywhere along the membrane equator; that is, we assumed that $w_{z}=w_{y}$ in the focal plane and that the two waist dimensions could be freely interchanged. This is not strictly true in the case of a linearly polarized excitation beam.

Suffice it to say that a full polarization analysis of the SHG radiation from membranes remains rather complex owing to the many possible hyperpolarizability components that may be involved, as evidenced by Eqs. (18)(20). Such a comprehensive analysis is beyond the scope of this paper.

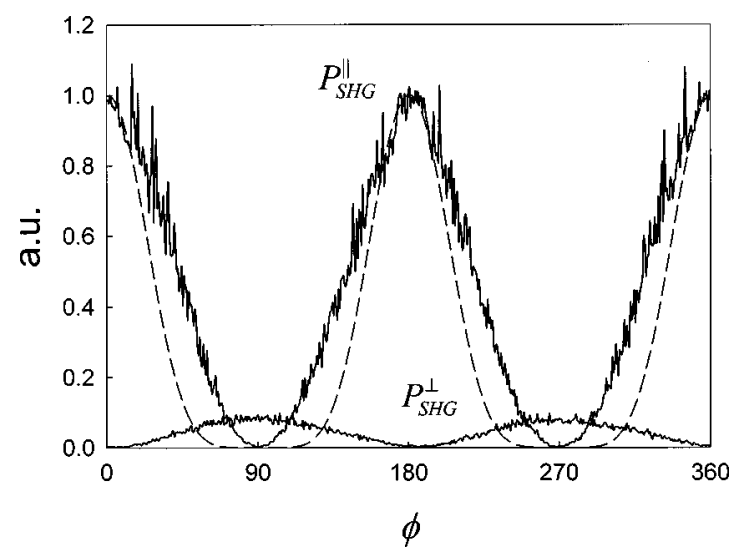

Fig. 7. Variation of SHG power along the equator of a GUV labeled with Di-6-ASPBS. Excitation is linearly polarized. A polarizer is inserted in the detection path and is oriented parallel $\left(P_{\mathrm{SHG}}^{\|}\right)$or perpendicular $\left(P_{\mathrm{SHG}}^{\perp}\right)$ to the excitation polarization. Plots are shown as a function of angle $\phi$ between the normal to the membrane plane ( $z$ axis) and the excitation polarization direction. The dashed trace corresponds to the $\cos ^{6} \phi$ dependence expected for perfectly aligned uniaxial molecules. 


\section{CONCLUSION}

Our purpose in this paper was to lay some theoretical foundations for high-resolution SHG imaging of membranes. Inasmuch as SHG imaging may be performed simultaneously with TPEF imaging, we have taken particular care to establish a formalism that permits direct comparison between the two modalities. Similarities between SHG and TPEF emission powers are that both scale with the square of the excitation intensity, that they may both be defined in terms of commensurate molecular cross sections, and that their active focal areas (or volumes) are independent of the sample size and depend only on the focus tightness. Differences lie in the fact that SHG power scales with the square of the number of molecules, whereas TPEF power scales only linearly with number. Moreover, a structure parameter is necessary for proper quantification of SHG power, which depends on the extent of the active focal area. We note finally that our formalism, which is essentially derived from regarding a distribution of hyperpolarizable molecules as a phased-array antenna, is readily extendible to the generation of harmonics of higher order. In all these cases, effective molecular cross sections may be rigorously defined, and an active focal area (or volume, as in the case of third-harmonic generation in bulk) may be readily identified.

\section{APPENDIX A}

A general theoretical framework for describing the electromagnetic field near the focus of a tightly focused beam has been proposed by Richard and Wolf. ${ }^{31}$ This framework, based on diffraction theory, may be applied to linear or nonlinear optical microscopy. In the case of TPEF microscopy, the signals are incoherent, and only the local field intensity, or correspondingly the field amplitude, need be calculated about the focal center. In the case of SHG microscopy, however, the signals are coherent, and the situation becomes more complicated. Both the local field amplitude and phase must be calculated about the focal center.

It is well known that any focused beam incurs a net $\pi$ phase shift after it passes through its focus. This phenomenon, commonly referred to as a phase anomaly, has a profound influence on the spatial structure of SHG radiation from a membrane. On closer inspection the phase shift, which is $\pi / 2$ exactly at the focal center, is found to vary approximately linearly with distance along the propagation axis near the focal center. As such, a focused beam behaves much as a plane wave near the focal center, though with an effective wave vector that has been modified relative to that of an unfocused beam. The effective wave vector of a focused beam near its focal center may be written as $\xi k_{\omega}$, where $k_{\omega}$ is the wave vector of a corresponding unfocused beam and in general $\xi$ is less than 1. Because the phase of the induced secondharmonic polarizability is governed by the phase of the excitation field, and because appreciable SHG power is produced only in directions that permit proper phase matching between this induced polarizability and the resultant SHG field, we find that, because $\xi<1$, the radi- ated SHG power in the forward $(\theta=0)$ direction is weak. The radiated SHG power along $\theta= \pm \theta_{\text {peak }}$, however, is appreciable because the fields in these directions are in phase and add coherently. Both $\theta_{\text {peak }}$ and the total radiated SHG power depend critically on $\xi$; hence a detailed evaluation of this parameter is warranted.

We use the integral formulas derived in Ref. 22 to examine how $\xi$ varies with the NA of the focusing objective of the excitation beam. To begin, we evaluate $\xi$ only at the focal center and consider the case $n_{2 \omega} \approx n_{\omega}$. We may define a focusing angle $\theta_{\mathrm{NA}}=\sin ^{-1}\left(\mathrm{NA} / n_{\omega}\right)$ to characterize the angular range of the excitation light. A comparison of $\theta_{\text {peak }}=\cos ^{-1}(\xi)$ and $\theta_{\mathrm{NA}}$ for several NA's is shown in Fig. 8. We observe that $\theta_{\text {peak }} / \theta_{\mathrm{NA}}$ is roughly constant and less than 1 , from which we obtain the general rule of thumb that a collection NA equal to the excitation NA is sufficient for essentially all the SHG light to be collected. In particular, for low NA's we find that

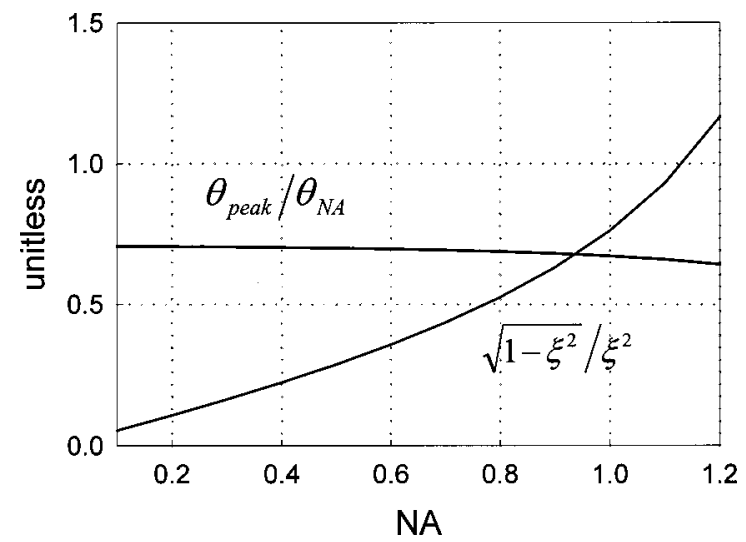

Fig. 8. Variations of the ratio $\theta_{\text {peak }} / \theta_{\mathrm{NA}}$ [where $\theta_{\mathrm{NA}}$ $\left.=\sin ^{-1}(\mathrm{NA} / n)\right]$ and of the parameter $\left(1-\xi^{2}\right)^{1 / 2} / \xi^{2}$ as a function of the NA of a water-immersion microscope objective, assuming a uniformly backfilled aperture and linear polarization. The plots are obtained from full numerical evaluations valid for arbitrary NA. For low NA, $\theta_{\text {peak }} \rightarrow \theta_{\mathrm{NA}} / \sqrt{2}$.

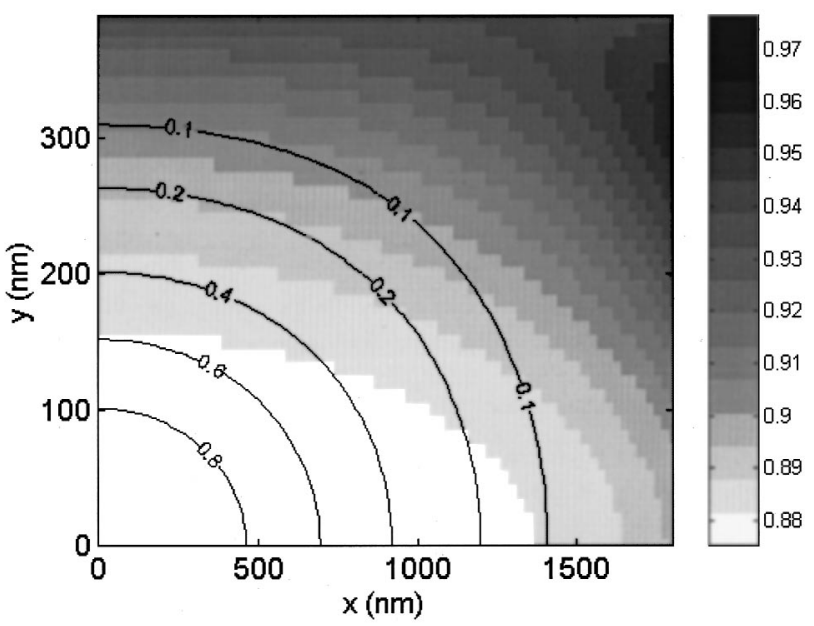

Fig. 9. Spatial profile of the phase-anomaly parameter $\xi$ (shades) and of the normalized intensity-squared distribution (contours) about the focal center $(0,0)$ of an 880-nm-wavelength laser beam focused by water-immersion objective with a NA of 0.9 . The axial propagation direction is $x$; the lateral direction is $y$. Profiles were calculated assuming a uniformly backfilled aperture and linear polarization. $\xi$ is relatively constant within the major portion of the intensity-squared profile. 
$\theta_{\text {peak }}=\theta_{\mathrm{NA}} / \sqrt{2}$. We also provide a plot of the parameter $\left(1-\xi^{2}\right)^{1 / 2} / \xi^{2}$ as found in expression (27). This parameter is found to vary linearly with NA for low to modest NA's.

Finally, throughout this paper we have assumed that $\xi$ is roughly constant over the entire membrane surface area that contributes to SHG radiation. To justify this assumption we illustrate the numerical evaluation of the $\xi(x, y)$ near the focal center and compare this with the corresponding intensity distribution of the excitation beam (Fig. 9) for our experimental case of interest (NA of 0.9 ). We observe that $\xi$ varies only slightly within the major portion of the intensity-squared distribution, and hence the approximation that $\xi$ is constant over the active surface area in which SHG is produced is quite reasonable. In our case, we find numerically that $\xi \approx 0.88$.

\section{ACKNOWLEDGMENTS}

The authors gratefully acknowledge financial support by the Insitut Curie and by the Centre National de Recherche Scientifique. We thank M. Blanchard-Desce for providing the dye molecules and S. Charpak and C. Boccara for helpful assistance. L. Moreaux was supported by a Bourse Docteur Ingénieur.

J. Mertz's e-mail address is jerome.mertz@espci.fr.

\section{REFERENCES}

1. W. Denk, J. H. Strickler, and W. W. Webb, "Two-photon laser scanning fluorescence microscopy," Science 248, 73-76 (1990).

2. J. R. Lakowicz and I. Gryczynski, "Multiphoton excitation of biochemical fluorophores," in Topics in Fluorescence Spectroscopy, J. R. Lakowicz, ed. (Plenum, New York, 1997), Vol. 5, p. 87.

3. C. Xu and W. W. Webb, "Multiphoton excitation of molecular fluorophores and nonlinear laser microscopy," in Topics in Fluorescence Spectroscopy, J. R. Lakowicz, ed. (Plenum, New York, 1997), Vol. 5, p. 471.

4. S. Maiti, J. B. Shear, R. M. Williams, W. R. Zipfel, and W. W. Webb, "Measuring serotonin distribution in live cells with three-photon excitation," Science 24, 530-532 (1997).

5. C. Xu, W. Zipfel, J. B. Shear, R. M. Williams, and W. W. Webb, "Multiphoton fluorescence excitation: new spectral windows for biological nonlinear microscopy," Proc. Natl. Acad. Sci. USA 93, 10,763-10,768 (1996).

6. J. I. Dadap, J. Shan, A. S. Weling, J. A. Misewich, A. Nahata, and T. F. Heinz, "Measurement of the vector character of electric fields by optical second-harmonic generation," Opt. Lett. 24, 1059-1061 (1999).

7. R. Gauderon, P. B. Lukins, and C. J. R. Sheppard, "Threedimensional second-harmonic generation imaging with femtosecond laser pulses," Opt. Lett. 23, 1209-1211 (1998).

8. Y. Guo, P. P. Ho, H. Savage, D. Harris, P. Sacks, S. Schantz, F. Liu, N. Zhadin, and R. R. Alfano, "Secondharmonic tomography of tissues," Opt. Lett. 22, 1323-1325 (1997).

9. G. Peleg, A. Lewis, M. Linial, and L. M. Loew, "Non-linear optical measurement of membrane potential around single molecules at selected cellular sites," Proc. Natl. Acad. Sci. USA 96, 6700-6704 (1999).

10. P. J. Campagnola, M. Wei, A. Lewis, and L. M. Loew,
"High-resolution nonlinear optical imaging of live cells by second harmonic generation," Biophys. J. 77, 3341-3349 (1999).

11. D. Yelin and Y. Silberberg, "Laser scanning thirdharmonic-generation microscopy in biology," Opt. Express 5, 169-175 (1999).

12. M. Muller, J. Squier, K. R. Wilson, and G. J. Brakenhoff, "3D-microscopy of transparent objects using thirdharmonic generation," J. Microsc. 191, 266-269 (1998).

13. T. F. Heinz, H. W. K. Tom, and Y. R. Shen, "Determination of molecular orientation of monolayer adsorbates by optical second-harmonic generation," Phys. Rev. A 28, 1883-1885 (1983).

14. N. Bloembergen, "Second harmonic reflected light," Opt. Acta 13, 311-322 (1966)

15. Y. R. Shen, The Principles of Nonlinear Optics (Wiley, New York, 1984).

16. R. Boyd, Nonlinear Optics (Academic, London, 1992).

17. A. Lewis, A. Khatchatouriants, M. Treinin, Z. Chen, G. Peleg, N. Friedman, O. Bouetvich, Z. Rothman, L. Loew, and M. Sheres, "Second-harmonic generation of biological interfaces: probing the membrane protein bacteriorhodopsin and imaging membrane potential around GFP molecules at specific sites in neuronal cells of $C$. elegans," Chem. Phys. 245, 133-144 (1999).

18. L. Moreaux, O. Sandre, M. Blanchard-Desce, and J. Mertz, "Membrane imaging by simultaneous second-harmonic generation and two photon microscopy," Opt. Lett. 25, 320-322 (2000).

19. A. Willets, J. E. Rice, D. Burland, and D. P. Shelton, "Problems in the comparison of theoretical and experimental hy perpolarizabilities," J. Chem. Phys. 97, 7590-7599 (1992).

20. C. Xu and W. W. Webb, "Measurement of two-photon excitation cross section of molecular fluorophores with data from $690 \mathrm{~nm}$ to $1050 \mathrm{~nm}$," J. Opt. Soc. Am. B 13, 481-491 (1996).

21. N. Bloembergen, Nonlinear Optics, 4th ed. (World Scientific, Singapore, 1965).

22. M. Born and E. Wolf, Principles of Optics, 6th ed. (Pergamon, Oxford, 1993).

23. J. Mertz, "Molecular photodynamics involved in multiphoton excitation fluorescence microscopy," Eur. Phys. J. D 3, 53-66 (1998).

24. S. R. Marder, D. N. Beratan, and L.-T. Cheng, "Approaches for optimizing the first hyperpolarizability of conjugated organic molecules," Science 252, 103-106 (1991).

25. T. Kogej, D. Beljonne, F. Meyers, J. W. Perry, S. R. Marder, and J. L. Brédas, "Mechanisms for enhancement of twophoton absorption in donor-acceptor conjugated chromophores," Chem. Phys. Lett. 298, 1-6 (1998).

26. D. S. Chemla and J. Zyss, Nonlinear Optical Properties of Organic Molecules and Crystals (Academic, New York, 1984).

27. J. L. Oudar, "Optical nonlinearities of conjugated molecules. Stilbene derivatives and highly polar aromatic compounds," J. Chem. Phys. 67, 446-457 (1977).

28. L. M. Loew and L. L. Simpson, "Charge shift probes of membrane potential. A probable electrochromic mechanism for ASP probes on a hemispherical lipid bilayer," Biophys. J. 34, 353-365 (1981).

29. O. Sandre, L. Moreaux, and F. Brochard, "Dynamics of transient pores in stretched vesicles," Proc. Natl. Acad. Sci. USA 96, 10,588-10,596 (1999).

30. C. W. Dirk, R. J. Twieg, and G. Wagniére, "The contribution of $\pi$ electrons to second harmonic generation in organic molecules," J. Am. Chem. Soc. 108, 5387-5395 (1986).

31. B. Richards and E. Wolf, "Electromagnetic diffraction in optical systems. II. Structure of the image field in aplanetic system,” Proc. R. Soc. London, Ser. A 253, 358-379 (1959). 